\title{
miR-504 promotes tumour growth and metastasis in human osteosarcoma by targeting TP53INP1
}

\author{
QINGCHUN CAI, SIXIANG ZENG, XING DAI, JUNLONG WU and WEI MA \\ Department of Orthopaedics, First Affiliated Hospital of the Medical College, \\ Xi'an Jiaotong University, Xi'an, Shaanxi 710061, P.R. China
}

Received March 18, 2017; Accepted September 4, 2017

DOI: $10.3892 /$ or.2017.5983

\begin{abstract}
An increasing number of studies have demonstrated that microRNAs participate in the development of osteosarcoma by acting as tumour suppressor or tumour-promoting genes. We investigated the role of miR-504 in the growth and metastasis of osteosarcoma. The expression of miR-504 in clinical osteosarcoma samples was higher than that in the adjacent normal tissue and correlated with tumour size and clinical stage. Tumour protein p53-inducible nuclear protein 1 (TP53INP1) was downregulated in the clinical osteosarcoma samples compared with the adjacent normal tissues and was consistently correlated with the clinical stage. The results of dual-luciferase reporter assay and western blot analysis demonstrated that the TP53INP1 gene is a direct target of miR-504. Altogether, the Cell Counting Kit-8 (CCK-8), the colony formation, the flow cytometry and the Transwell assay results demonstrated that miR-504 promoted osteosarcoma cell growth and metastasis in vitro. $\mathrm{P} 73, \mathrm{P} 21, \mathrm{Bax}$, cleaved-caspase-3 and secreted protein acidic and rich in cysteine (SPARC) were associated with the suppressive role of miR-504/TP53INP1. The overexpression of miR-504 in osteosarcoma xenografts enhanced the tumour growth and increased the metastatic burden. Collectively, these results revealed that TP53INP1 is a target gene of miR-504 and that miR-504 enhances osteosarcoma growth and promotes distant metastases by targeting TP53INP1. Thus, miR-504/TP53INP1 may be associated with osteosarcoma size and clinical stage.
\end{abstract}

\section{Introduction}

Osteosarcoma is one of the most common primary bone tumours, affecting patients of all ages, however there are two incidence peaks. The first peak is in childhood and adolescense

Correspondence to: Professor Wei Ma, Department of Orthopaedics, First Affiliated Hospital of the Medical College, Xi'an Jiaotong University, 277 Yanta West Street, Xi'an, Shaanxi 710061, P.R. China E-mail: mawei60@126.com

Key words: osteosarcoma, miR-504, TP53INP1 and the second peak is at $75-79$ years of age $(1,2)$. The primary tumour originates in the distal femur, proximal tibia or humerus and its rapid cell division may contribute to the oncogenesis of osteosarcoma during childhood (3). Adjuvant chemotherapy following surgery, has improved the long-term survival rates from $<20$ to $70 \%$ in localized osteosarcoma. However, this combined therapy has shown limited progress in the long-term survival rates of metastatic osteosarcoma cases, which account for one-quarter of all patients at the time of initial diagnosis. The higher incidence of osteosarcoma in children and adolescents, its tendency to metastasize and the poor prognosis in metastatic cases render osteosarcoma the leading cause of cancer-related deaths in childhood, although it accounts only for $5 \%$ of childhood cancers (4). A better understanding of tumour initiation, metastasis and recurrence in osteosarcoma is urgently needed to improve patient prognosis.

Mature microRNAs (miRNAs) are endogenic regulatory, single-stranded, non-coding RNAs that are $22 \mathrm{nt}$ in length. miRNAs regulate one-third of all genes by complete or partial base-pairing at the 3'-untranslated region of their target mRNAs, resulting in translation inhibition and/or cleavage $(5,6)$. Thus, miRNAs can serve as either oncogenes or tumour suppressors depending on their target genes $(7,8)$. Accumulating evidence indicates that some miRNAs are differentially expressed in osteosarcoma tissues, cell lines and patient sera and that these miRNAs play important roles in tumorigenesis, metastasis and angiogenesis $(9,10)$. These findings have provided new insights into the genetic mechanisms of osteosarcoma tumorigenesis and have contributed to the identification of novel osteosarcoma biomarkers and the development of miRNA-targeted treatments (11).

miR-504 has rarely been studied and not much is known about its roles in oncogenesis and cancer metastasis. Computational prediction for potential targets of miR-504 indicated that this miRNA may directly interact with the mRNAs of FOXP1, NRF1, CDK6, P53 and tumour protein p53-inducible nuclear protein 1 (TP53INP1) genes. Recent studies demonstrated that miR-504 directly targets the FOXP1, NRF1, CDK6 and P53 genes (12-15). TP53INP1 was demonstrated to regulate the transcriptional activity of P53 or P73 through direct interaction. Furthermore, TP53INP1 participates in autophagy by binding LC3, PINK1 and Parkin (16). Notably, TP53INP1 and P53 are functional partners because TP53INP1 is a target gene of the transcription factor P53. 
In return, TP53INP1 can activate the transcription activity of P53 (17). To avoid interference among miR-504, P53 and TP53INP1, the P53-null osteosarcoma cell line 143B (IARC TP53 database, http://p53.iarc.fr/Manual.aspx) was used to explore the influence of miR-504/TP53INP1 modulation on tumour growth and metastasis in vitro and in vivo.

\section{Materials and methods}

Statement of ethics. All procedures involving human participants were performed in accordance to the 1964 Declaration of Helsinki and its later amendments. The animal research protocol was approved by the Animal Experiment Centre Committee of Xi'an Jiaotong University. All animal experimental procedures were performed according to the policy of Xi'an Jiaotong University Animal Experiment Centre.

Clinical samples and cell line. A total of 20 pairs of primary osteosarcoma and matched adjacent non-cancerous paraffin-embedded specimens were obtained at the authors' institution between 2012 and 2015. The osteosarcoma diagnosis and the confirmation that the adjacent tissues were normal were performed by two independent pathologists based on the pathological changes associated with osteosarcoma and the microscopic morphology of the adjacent normal tissues. The tumour stage was assessed according to the Enneking-Musculoskeletal Tumour Staging System (18). The human osteosarcoma cell line 143B was purchased from the Type Culture Collection of the Chinese Academy of Science, (Wuhan, China) and was cultured in Dulbecco's modified Eagle's medium (DMEM; Gibco, Grand Island, NY, USA) supplemented with $10 \%$ foetal bovine serum (FBS; Gibco) and $0.015 \mathrm{mg} / \mathrm{ml}$ 5-bromo-2'-deoxyuridine (Sigma-Aldrich, St. Louis, MO, USA) in a humidified incubator at $37^{\circ} \mathrm{C}$ with $5 \% \mathrm{CO}_{2}$.

Modulation of miR-504 in vitro. miR-504 mimics or inhibitors (RiboBio, Guangzhou, China) were transfected into 143B cells with the Ribo FECT ${ }^{\mathrm{TM}}$ CP Transfection kit (all reagents from RiboBio) according to the manufacturer's instructions to achieve the overexpression or knockdown of miR-504, respectively. Using the Ribo FECT ${ }^{\text {Tм }}$ CP Transfection kit, a negative control mimic (normal control) (RiboBio) and siR-Ribo ${ }^{\mathrm{TM}}$ Transfection Control (Cy3; RiboBio) were transfected into 143B cells as the normal and transfection controls, respectively.

RNA preparation and quantitative real-time PCR. The microRNAs were extracted from the clinical samples and cell lines using the miRNAprep Pure FFPE kit (Tiangen Biotech, Beijing, China) or the TRIzol reagent (Invitrogen, Carlsbad, CA, USA) according to the manufacturer's instructions. The miRcute miRNA First-Strand cDNA Synthesis kit and miRcute miRNA qPCR Detection kit (SYBR Green; Tiangen Biotech) were used for cDNA synthesis and RT-qPCR according to the manufacturer's instructions. The RT-qPCR conditions were $95^{\circ} \mathrm{C}$ for 5 min followed by 40 cycles of $95^{\circ} \mathrm{C}$ for $15 \mathrm{sec}$ and $60^{\circ} \mathrm{C}$ for $20 \mathrm{sec}$ and a final dissociation stage. The PCR was performed using the Roche LightCycler ${ }^{\circledR} 96$ fluorescent quantitative PCR instrument. The relative miR-504 expression level was determined using the $2^{-\Delta \mathrm{Ct}}$ method with the nuclear U6 RNA as the endogenous control (19). The forward primers were designed as follows: miR-504, 5'-GACCCTGGTCTGCACTCTATCA-3'; and U6, 5'-ACGCAAATTCGTGAAGCGTTC-3'. The reverse primers were provided with the kit.

Luciferase reporter system. The potential targets of miR-504 were predicted by miRanda (http://www.microrna.org). The predicted miR-504 binding site at the TP53INP1 3'-UTR and corresponding mutant 3'-UTR sequence were cloned in the psiCHECK $^{\mathrm{TM}}-2$ Vector (Promega, Madison, WI, USA). The psiCHECK-2-TP53INP1-WT and psiCHECK-2-TP53INP1MT were co-transfected with miR-504 NC or miR-504 mimic into $293 \mathrm{~T}$ cells. The relative luciferase activity was analysed using a Promega Dual-Luciferase system (Promega) after $48 \mathrm{~h}$ of transfection.

Colony formation assay. Four hundred cells were seeded in 60-mm dishes and cultured for nine days. Subsequently, the medium was removed. The colonies were fixed with $4 \%$ paraformaldehyde for $10 \mathrm{~min}$, stained with $1 \%$ crystal violet for $15 \mathrm{~min}$ and then counted.

Cell proliferation assay. The cells were transfected and seeded in 96-well plates at a density of 1,000 cells/well. The medium was changed every three days. The cells were harvested each day from the third to the seventh day and the cell viability was detected with Cell Counting Kit-8 (Beyotime Institute of Biotechnology, Shanghai, China) according to the manufacturer's instructions.

Cell cycle and apoptosis assay. The transfected cells were stained with $50 \mu \mathrm{g} / \mathrm{ml}$ propidium iodide (Sigma-Aldrich) and $250 \mu \mathrm{g} / \mathrm{ml}$ RNAase (Sigma-Aldrich). After 30 min of incubation in the dark, the cell cycle was analysed using a FACSCalibur flow cytometer (BD Biosciences, Franklin Lakes, NJ, USA). Cell apoptosis was analysed using an Annexin V-FITC/PI Apoptosis Detection kit (Wanleibio, Shenyang, China) by flow cytometry according to the manufacturer's instructions.

Cell migration and invasion assay. To determine the cell migration, $3 \times 10^{4}$ cells were reseeded in the top of a Transwell chamber $48 \mathrm{~h}$ after transfection. The cells that remained in the upper chamber after $24 \mathrm{~h}$ were carefully removed using a cotton swab. The migrated cells were fixed and stained with $1 \%$ crystal violet for $20 \mathrm{~min}$. The procedure for the cell invasion was similar to the migration assay, except for the following two differences: the upper Transwell chamber was precoated with $60 \mu \mathrm{l}$ of diluted Matrigel (BD Biosciences) and incubated at $37^{\circ} \mathrm{C}$ overnight with $6 \times 10^{4}$ transfected cells seeded in the upper chamber.

Western blot analysis. Seventy-two hours after transfection, the total cellular protein was extracted using RIPA lysis buffer and Phosphatase Inhibitor II Cocktail (50X) (Heart, Xi'an, China) according to the manufacturer's instructions. The total protein was separated and transferred onto polyvinylidene membranes (Merck Millipore, Billerica, MA, USA). Subsequently, the membranes were blocked and incubated with primary antibodies overnight at $4^{\circ} \mathrm{C}$. Following incubation 
Table I. Relationship between the expression of miR-504, TP53INP1 and the clinical characteristics in 20 osteosarcoma patients.

\begin{tabular}{|c|c|c|c|c|c|c|}
\hline \multirow{2}{*}{$\begin{array}{l}\text { Clinical } \\
\text { characteristics }\end{array}$} & \multirow[b]{2}{*}{$\mathrm{N}$} & \multirow{2}{*}{$\begin{array}{l}\text { miR-504 } \\
\text { expression }\end{array}$} & \multirow[b]{2}{*}{ P-value } & \multicolumn{2}{|c|}{ TP53INP1 expression } & \multirow[b]{2}{*}{ P-value } \\
\hline & & & & Positive & Negative & \\
\hline \multicolumn{7}{|l|}{ Sex } \\
\hline Female & 8 & $2.069 \pm 0.896$ & 0.630 & 4 & 4 & 0.535 \\
\hline Male & 12 & $1.676 \pm 0.284$ & & 5 & 7 & \\
\hline \multicolumn{7}{|l|}{ Age (years) } \\
\hline$<18$ & 9 & $2.254 \pm 0.766$ & 0.336 & 3 & 6 & 0.311 \\
\hline$\geq 18$ & 11 & $1.488 \pm 0.319$ & & 6 & 5 & \\
\hline \multicolumn{7}{|l|}{ Tumour size $(\mathrm{cm})$} \\
\hline$<10$ & 13 & $1.251 \pm 0.235$ & $<0.05$ & 7 & 6 & 0.272 \\
\hline$\geq 10$ & 7 & $2.914 \pm 0.914$ & & 2 & 5 & \\
\hline \multicolumn{7}{|l|}{ Clinical stage } \\
\hline IA-IIA & 7 & $0.793 \pm 0.138$ & $<0.05$ & 6 & 1 & $<0.05$ \\
\hline IIB-III & 13 & $2.393 \pm 0.530$ & & 3 & 10 & \\
\hline \multicolumn{7}{|l|}{ Distal metastasis } \\
\hline Yes & 7 & $3.125 \pm 0.912$ & $<0.05$ & 1 & 6 & 0.058 \\
\hline No & 13 & $1.137 \pm 0.149$ & & 8 & 5 & \\
\hline \multicolumn{7}{|l|}{ Tumour site } \\
\hline Femur or tibia & 14 & $1.984 \pm 0.509$ & 0.563 & 5 & 9 & 0.217 \\
\hline Other & 6 & $1.481 \pm 0.519$ & & 4 & 2 & \\
\hline \multicolumn{7}{|l|}{ TP53INP1 } \\
\hline Positive & 9 & $0.778 \pm 0.106$ & $<0.05$ & & & \\
\hline Negative & 11 & $2.696 \pm 0.582$ & & & & \\
\hline
\end{tabular}

TP53INP1, tumour protein p53-inducible nuclear protein 1.

with an HRP-conjugated goat anti-rabbit IgG secondary antibody (dilution, 1:5,000; Boster Biological Technology, Wuhan, China) at room temperature for $1 \mathrm{~h}$, the protein signals were detected using the Immobilon ${ }^{\mathrm{TM}}$ Western Chemiluminescent HRP Substrate (Merck Millipore). The following primary antibodies were used: rabbit anti-TP53INP1 (dilution, 1:2,000; Abcam, Cambridge, MA, USA), rabbit anti-P53 (phospho S46; dilution, 1:1,000; Abcam), rabbit anti-GAPDH (dilution, 1:1000; Wanleibio), rabbit anti-P53 (dilution, 1:800; Wanleibio), rabbit anti-P73 (dilution, 1:500; Wanleibio), rabbit anti-P21 (dilution, 1:500; Wanleibio), rabbit anti-Bax (dilution, 1:500; Wanleibio), rabbit anti-caspase-3 (dilution, 1:500; Wanleibio), rabbit anti-cleaved-caspase-3 (dilution, 1:300; Wanleibio) and rabbit anti-SPARC (dilution, 1:1000; Sanying Biotechnology, Wuhan, China).

Xenografts in nude mice and associated histopathology. Ten five-week-old nude mice were randomly and equally divided into the miR-504 agomir and the miR-504 NC groups. Subsequently, $3 \times 10^{6}$ cells were subcutaneously injected in the flanks of the nude mice. The cells had been pre-transfected with agomir-hsa-miR-504 (miR-504 agomir; GenePharma, Shanghai, China) or agomir negative control (miR-504 NC; GenePharma) based on the grouping. One week after the tumour-cell implantation, $1 \mathrm{nmol}$ of miR-504 agomir or $1 \mathrm{nmol}$ of agomir miR-504 $\mathrm{NC}$ was injected into the tumours twice a week; the tumour volume was concurrently assessed according to the formula: tumour volume = length $\mathrm{x}$ width ${ }^{2} / 2$. All mice were sacrificed four weeks after inoculation and orthotopic tumours and lungs were harvested. After weighing the orthotopic tumours, both tumours and lungs were stained with haematoxylin and eosin (H\&E).

Immunohistochemistry. The slides containing formalin-fixed, paraffin-embedded, 5- $\mu \mathrm{m}$ thick tissue sections were subjected to microwave antigen retrieval and then incubated with the primary antibody overnight at $4^{\circ} \mathrm{C}$. Subsequently, the slides were incubated with an HRP-conjugated secondary antibody for $30 \mathrm{~min}$ at room temperature and the proteins were chemically visualised using a DAB kit (ZSGB-Bio, Shanghai, China).

Statistical analysis. Statistical comparisons of the results were performed using the SPSS 15.0 software (SPSS Inc., Chicago, IL, USA) with Student's t-test, independent-samples t-test or Fisher's exact probability test, depending on the particular data type. $\mathrm{P}<0.05$ was considered to indicate a statistically significant difference.

\section{Results}

miR-504 is highly expressed in the osteosarcoma tissues. Expression of miR-504 was higher in 17 osteosarcoma 

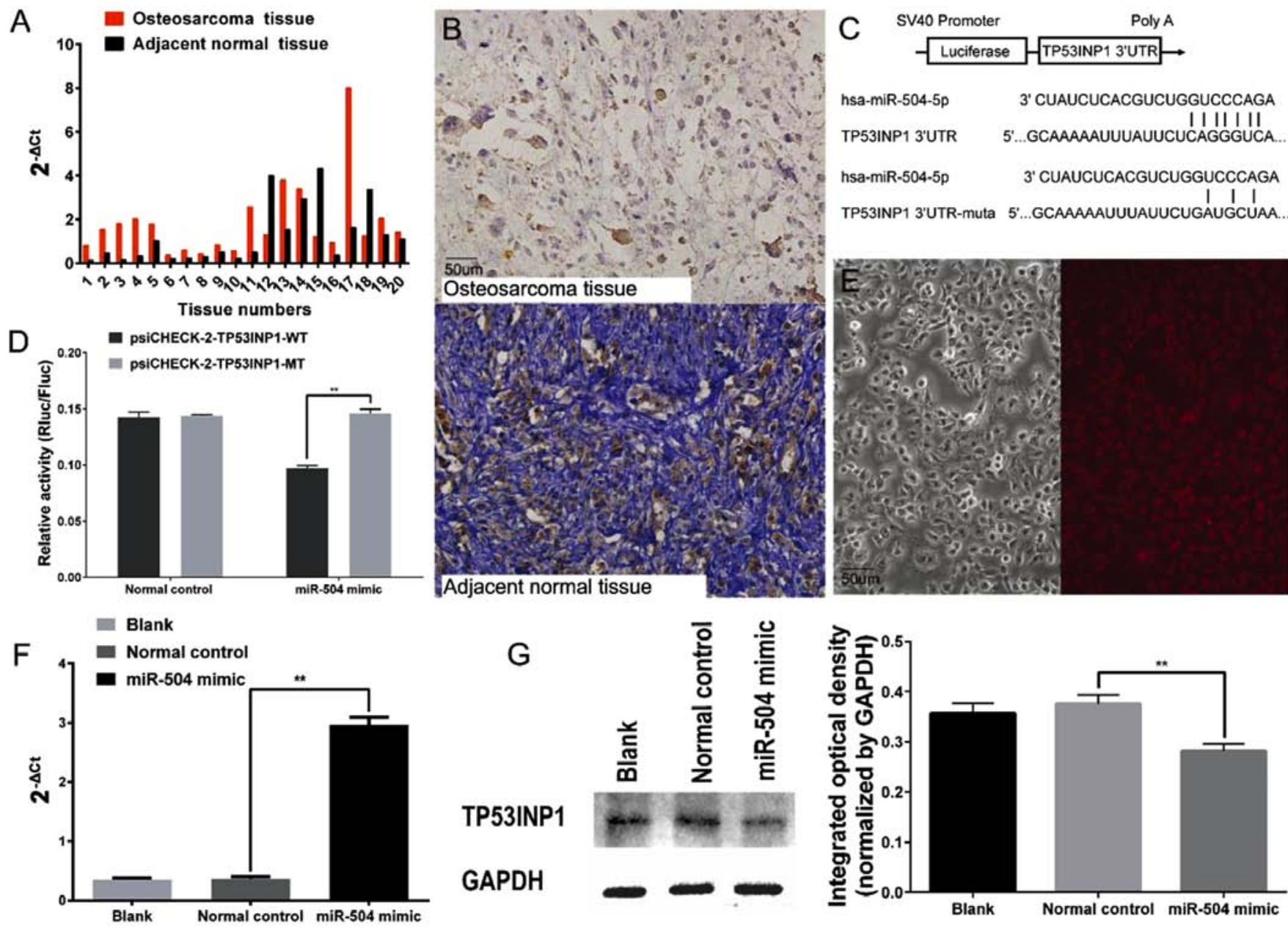

Figure 1. Expression of miR-504/TP53INP1 in clinical samples and direct targeting of TP53INP1 by miR-504. (A) miR-504 expression was higher in the osteosarcoma tissues than in the adjacent normal tissues (as determined by RT-qPCR). (B) TP53INP1 expression in the osteosarcoma tissues and adjacent normal tissues was determined by immunohistochemical staining. (C) The predicted binding sequence and paired mutated sequence were inserted into the luciferase reporter vector. (D) Relative luciferase activity in 293T cells co-transfected with the psiCHECK-2-TP53INP1-WT or psiCHECK-2-TP53INP1-MT plasmid and the miR-504 mimic or the normal control. The experiment was performed in triplicate. (E) 143B cells showing red fluorescence after transfection with the Cy3-labelled NC siRNA. (F) miR-504 expression in 143B cells transfected with the miR-504 mimic or the normal control (as determined by RT-qPCR). The experiment was performed in triplicate. (G) TP53INP1 expression in 143B cells transfected with the miR-504 mimic or the normal control (as determined by western blotting). The experiment was performed in triplicate. ${ }^{* * *} \mathrm{P}<0.01$ vs. the normal control. TP53INP1, tumour protein p53-inducible nuclear protein 1 .

specimens compared to the matched adjacent non-cancerous tissues and the remaining tumour specimens had a relatively lower expression of miR-504 based on RT-qPCR assay (Fig. 1A). As shown in Table I, miR-504 was more highly expressed in patients with distant metastasis, advanced clinical stage and larger tumours. Additionally, TP53INP1positive specimens had a statistically lower expression of miR-504. There was no significant difference between miR-504 expression and age or sex.

TP53INP1 is not frequently expressed in osteosarcoma tissues. TP53INP1 was detected in nine osteosarcoma specimens (45\%) using immunohistochemical staining, which was tan-coloured and located in the nucleus. Seventeen adjacent non-cancerous specimens (85\%) exhibited TP53INP1 expression (Fig. 1B). Furthermore, the detectable expression of TP53INP1 was infrequent in patients with advanced clinical stage according to Fisher's exact probability test (Table I).
miR-504 directly targets TP53INP1. We searched for potential targets of miR-504 using the miRanda databases and found a potential miR-504-binding site in the 3'UTR of the TP53INP1 mRNA. To validate the direct binding between miR-504 and the predicted seed sequence, the predicted potential binding sequence and paired mutated sequence were inserted into the psiCHECK ${ }^{\mathrm{TM}}-2$ vector (Fig. 1C). Luciferase activity was significantly decreased after co-transfection of psiCHECK-2-TP53INP1-WT and miR-504 mimic, whereas miR-504 had no effect on luciferase activity when co-transfected with psiCHECK-2-TP53INP1-MT (Fig. 1D). The feasibility of the transfection was verified using Cy3-labelled normal control siRNA and RT-qPCR in 143B cells (Fig. 1E and F). Furthermore, the results of western blot analysis confirmed that the expression of TP53INP1 was downregulated in the 143B cells after the miR-504 mimic transfection (Fig. 1G).

miR-504 promotes $143 B$ cell growth, migration and invasion in vitro. The CCK-8 assay was performed to investigate 

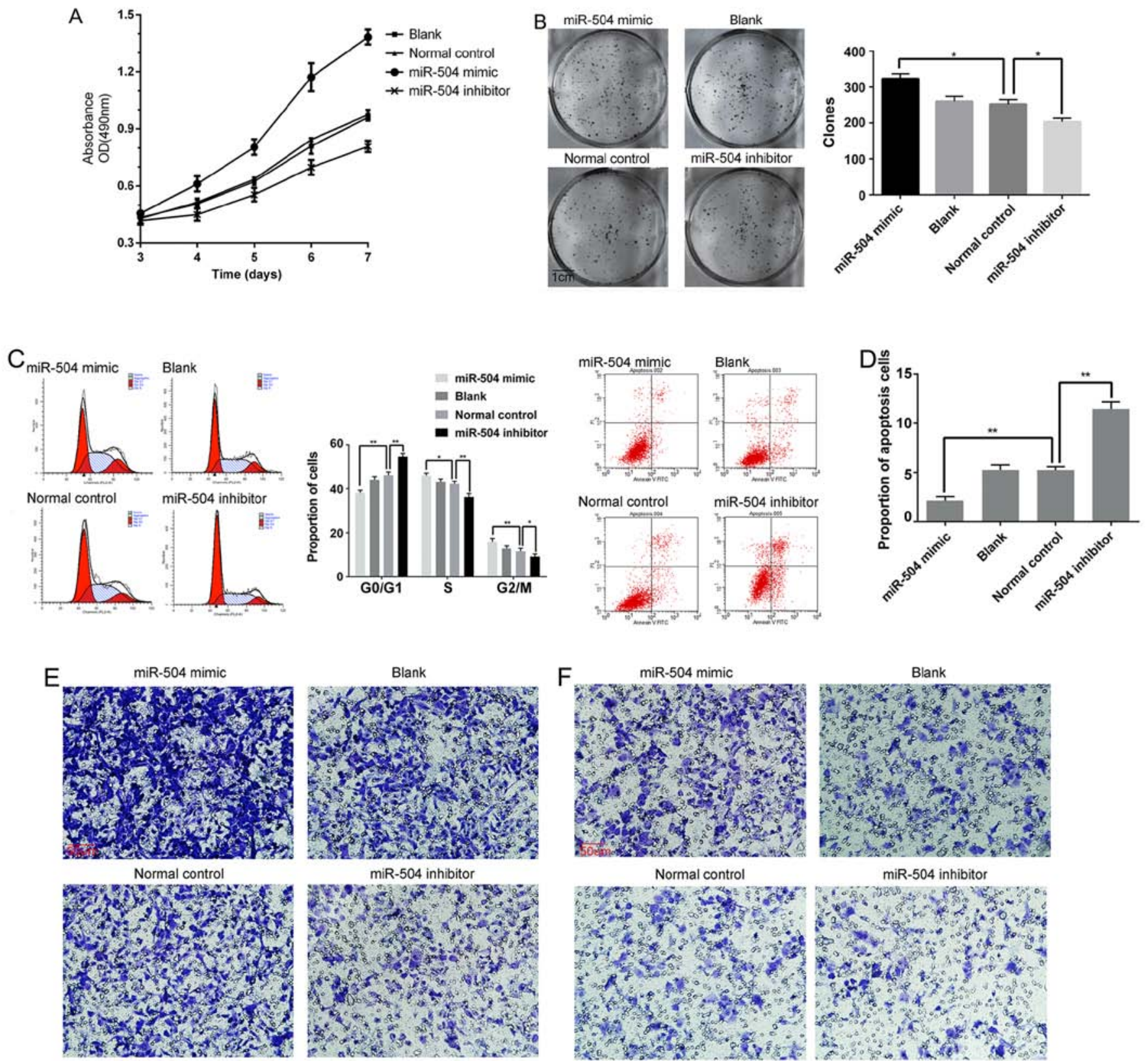

Figure 2. miR-504 enhances 143B cell growth and promotes metastasis in vitro by targeting TP53INP1. (A) The Cell Counting Kit- 8 assay was performed to evaluate cell proliferation 3-7 days post-transfection. (B) The clone formation assay was performed to examine cell activity at 9 days post-transfection. (C) The cell cycle distribution was determined by flow cytometric analysis with propidium iodide staining. (D) The proportion of apoptotic cells was determined by flow cytometric analysis with Annexin-V and propidium iodide staining. (E) Transwell assay results. The images show the cells that travelled through the micropore membrane without Matrigel. (F) Transwell assay results. The images show the cells that travelled through the micropore membrane with Matrigel. Normal control, 143B cells transfected with the normal control; miR-504 mimic, 143B cells transfected with the miR-504 mimic; miR-504 inhibitor, 143B cells transfected with the miR-504 inhibitor. ${ }^{*} \mathrm{P}<0.05,{ }^{* *} \mathrm{P}<0.01$ vs. the normal control. All experiments were performed in triplicate. TP53INP1, tumour protein p53-inducible nuclear protein 1.

the effects of miR-504 on the proliferation of 143B cells. Proliferation was significantly enhanced in 143B cells transfected with miR-504 mimic and decreased with miR-504 inhibition compared with the normal control and blank groups (Fig. 2A). Consistently, the cells transfected with the miR-504 mimic had more colonies in the colony formation assay, whereas fewer colonies survived in the normal control and blank groups. The lowest number of colonies was found in the miR-504 inhibitor group (Fig. 2B).

Based on a cell cycle assay, the G1 subpopulation proportion of cells was decreased in the miR-504 mimic-transfected cells compared with other groups and the proportion of cells in the G2 subpopulation was increased. In contrast, G1 arrest was obvious in the cells transfected with the miR-504 inhibitor, which blocked the G1 to S-phase transition, compared with the blank and normal control groups (Fig. 2C). The transfection of miR-504 mimic mitigated apoptosis in the 143B cells compared with the normal control and blank groups and the cells transfected with the miR-504 inhibitor had the highest proportion of apoptosis (Fig. 2D).

To investigate the effects of miR-504 on migration and invasion in 143B cells, a Transwell assay was performed. 


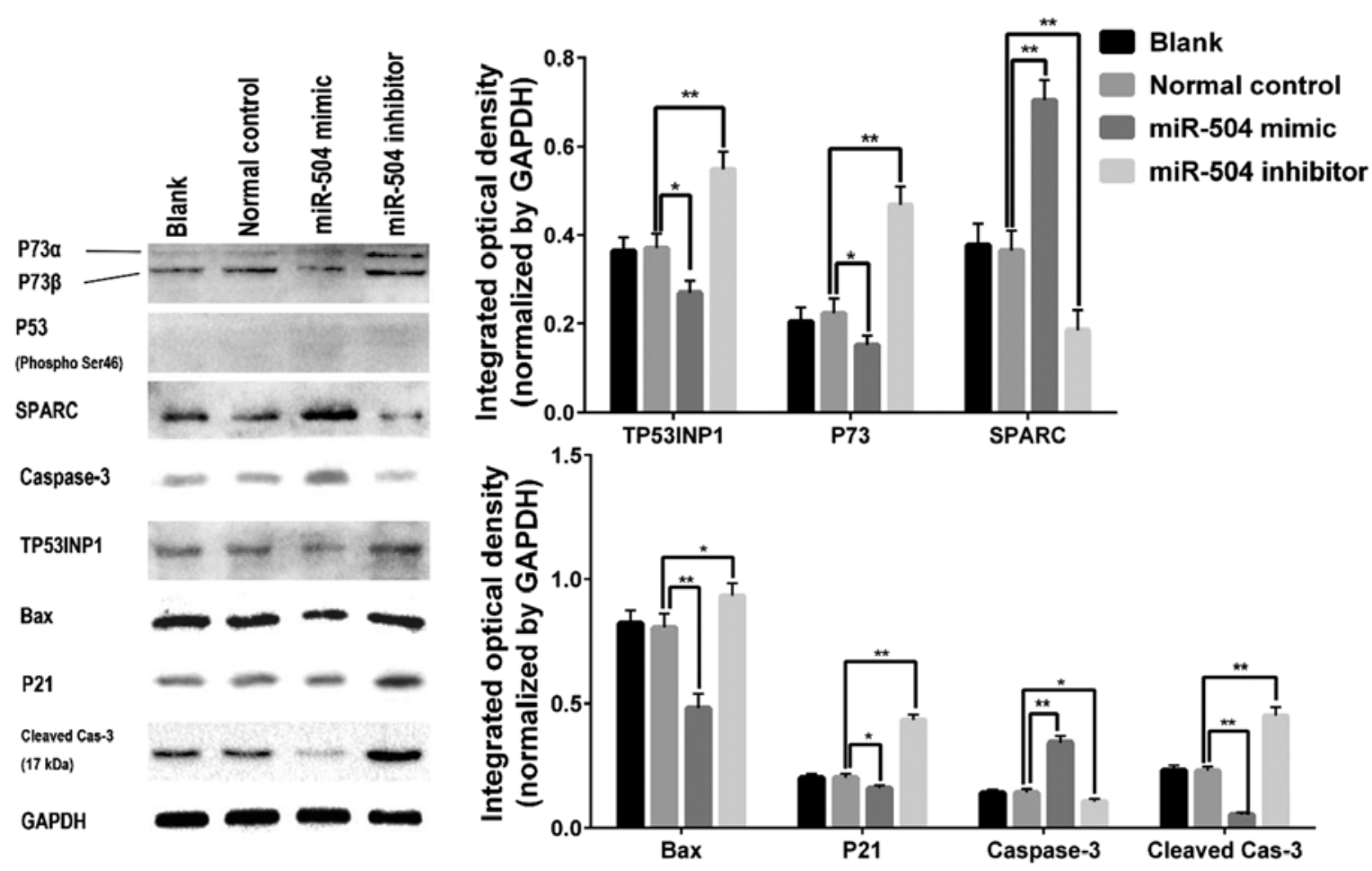

Figure 3. Western blot analysis examining the expression of TP53INP1, phosphorylated P53, SPARC, P73 and its downstream proteins in response to miR-504. Normal control, 143B cells transfected with the normal control; miR-504 mimic: 143B cells transfected with the miR-504 mimic; miR-504 inhibitor, 143B cells transfected with the miR-504 inhibitor. ${ }^{*} \mathrm{P}<0.05,{ }^{* *} \mathrm{P}<0.01$ vs. the normal control. The experiment was performed in triplicate. TP53INP1, tumour protein p53-inducible nuclear protein 1; SPARC, secreted protein acidic and rich in cysteine.
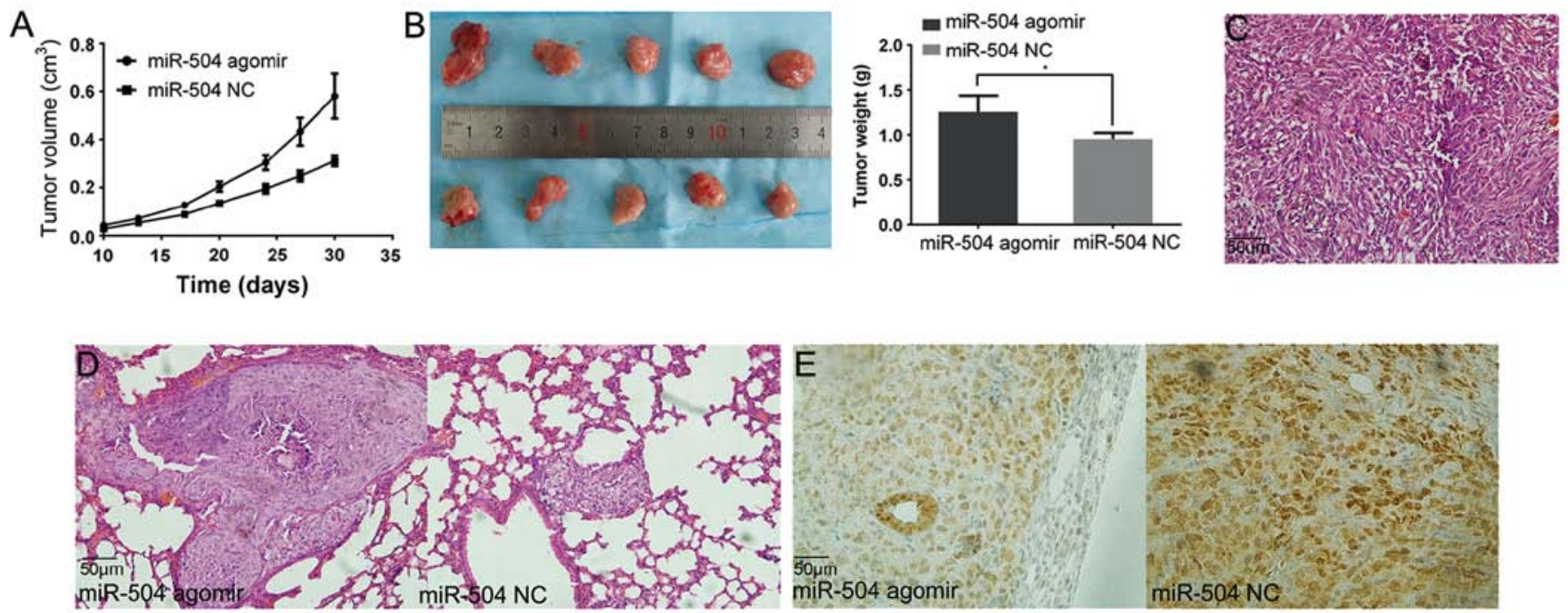

Figure 4. The miR-504 agomir promotes tumour growth and distant metastasis in vivo. (A) Nude mice were subcutaneously transplanted with $143 \mathrm{~B}$ cells transfected with the miR-504 normal control or the miR-504 agomir. The tumour volume was calculated twice weekly after injection of the miR-504 agomir and the miR-504 normal control. The tumour volume was calculated as follows: length $\mathrm{x}$ width ${ }^{2} \mathrm{x} 0.5$. The tumour volumes were assessed in triplicate. (B) Mice were sacrificed four weeks after implantation. The tumours (top of the Vernier calliper) in the miR-504 agomir-treated group were larger and heavier. The tumour weights were assessed in triplicate. (C) H\&E staining showing the microscopic morphology of the harvested tumours. (D) H\&E staining showing the microscopic morphology of a metastatic node in the lung. (E) TP53INP1 expression in the xenograft tumours was determined by immunohistochemical staining. " $\mathrm{P}<0.05$ vs. the miR-504 normal control. H\&E, haematoxylin and eosin; TP53INP1, tumour protein p53-inducible nuclear protein 1.

Cells in the miR-504 mimic group exhibited a strengthened migration and invasion capacity compared with the normal control and blank groups, while minimal numbers of cells traversed the polycarbonate membrane of the Transwell chamber in the miR-504 inhibitor group for both the migration and invasion assays (Fig. 2E and F).
Finally, we evaluated TP53INP1, phosphorylated P53, P73, P21, Bax, cleaved-caspase-3 and SPARC expression and found that TP53INP1, P73, P21, Bax and cleaved-caspase-3 expression was decreased $72 \mathrm{~h}$ after the miR-504 mimic transfection, but upregulated in the miR-504 inhibitor group. SPARC is correlated with pancreatic cancer cell migration 
and was upregulated in 143B cells after the miR-504 mimic transfection compared with the normal control and blank groups. However, phosphorylated P53 was undetectable in all four groups (Fig. 3).

miR-504 promotes xenograft growth and distant metastasis in vivo. The tumour growth was faster in the miR-504 agomir group than in the miR-504 NC group (Fig. 4A). The tumours were harvested and weighed at the fourth week and the tumour weights ascertained faster growth in the miR-504 agomir group (1.29 vs. 0.95 g) (Fig. 4B). The microscopical morphology of the tumour was also visualised using H\&E staining (Fig. 4C).

All lungs were harvested and stained with $\mathrm{H} \& \mathrm{E}$ to detect distal metastases. Only one mouse developed pulmonary metastasis in the miR-504 NC group and three mice developed pulmonary metastasis in the miR-504 agomir group. Mice in the latter group developed larger metastatic tumours (Fig. 4D).

Although all tumours had detectable TP53INP1 expression, the expression level was lower in the miR-504 agomir-treated tumours (Fig. 4E).

\section{Discussion}

Many studies have revealed that miRNAs serve as oncogenes or tumour suppressors in various types of cancer depending on the particular miRNA (20). miR-504 has been demonstrated to participate in tumour development and metastasis by targeting FOXP1, NRF1 and P53 in several tumours, acting as an oncogene $(13,15,21,22)$. Clinical samples demonstrated that higher expression levels of miR-504 are correlated with worse radio-therapeutic effects in nasopharyngeal carcinoma and with a poor prognosis for pancreatic ductal adenocarcinoma patients $(13,23)$. In the present study, the expression of miR-504 was higher in most clinical osteosarcoma samples compared to adjacent non-cancerous tissues, which statistically correlated with tumour size, metastasis and clinical stage. Similar to the expression of miR-504, TP53INP1 was negatively correlated with the clinical stage and the expression of TP53INP1 was almost non-existent in patients with distant metastases.

One miRNA can regulate hundreds of proteins and one protein can be regulated by multiple miRNAs. These complex interactions between miRNAs and target proteins constitute a regulatory network $(24,25)$. The miRNA target prediction database provides thousands of potential target proteins for one particular miRNA. With the assistance of the luciferase reporter system, RT-qPCR and western blotting, only a few proteins have been demonstrated to be directly regulated by a particular miRNA. TP53INP1 is a computationally analysed target of miR-504. The luciferase reporter assay system confirmed a direct interaction of the miR-504 mimic with the predicted seed region in the TP53INP1 3'-UTR. Moreover, the western blot analysis results demonstrated lower TP53INP1 expression in the osteosarcoma 143B cells transfected with the miR-504 mimic than in the control cells, which further ascertained the direct regulation between miR-504 and TP53INP1 in $143 \mathrm{~B}$ cells.

Several studies have revealed that TP53INP1 functions as a tumour suppressor through P53 phosphorylation at serine-46 by forming a protein complex with the protein kinase homeodomain-interacting protein kinase-2 (HIPK2) or protein kinase $\mathrm{C} \delta(\mathrm{PKC} \delta$ ) to induce $\mathrm{G} 1$ arrest and apoptosis $(17,26,27)$. Because of the high degree of structural similarity with P53, P73 activates the transcription activity of the majority of P53-responsive genes and serves as a tumour suppressor. These responsive genes include P21 and Bax, as reported by Tomasini et al (28). Importantly, P73 is more stable in tumours than P53, which is often mutated $(29,30)$. Due to a missense mutation at exon 5, TP53 is inactive in the osteosarcoma 143B cell line and serine-46-phosphorylated P53 was undetectable in all four groups, confirming the loss-offunction mutation of P53 in 143B cells. In the present study, miR-504 promoted proliferation and mitigated apoptosis in 143B cells in vitro. Additionally, larger xenograft tumours were detected in nude mice with increased expression of miR-504. Furthermore, protein detection revealed that TP53INP1, P73, $\mathrm{P} 21$ and Bax were suppressed by miR-504. Both P73 $\alpha$ and $\mathrm{P} 73 \beta$, which promote tumour apoptosis, were downregulated after the miR-504 transfection (31). Phosphorylation, acetylation, ubiquitylation and sumoylation have been demonstrated to occur and to determine the stabilization and transactivation ability of P73. TP53INP1 has been shown to stimulate the cell cycle arrest and pro-apoptotic functions of P73, however the specific underlying mechanism remains unknown (28,32-35). The variation in protein expression further confirms the results of the cell cycle and apoptosis analyses. In conclusion, miR-504 promotes tumour growth through TP53INP1, which partly depends on suppressing P73 and downstream proteins and this effect occurs independently of P53 in 143B cells. The present study also reveals that miR-504 inhibition further inhibits tumour growth.

The suppression of TP53INP1 promotes tumour metastasis in endometrial carcinoma and non-small cell lung cancer. TP53INP1 also participates in decreasing the epithelial-mesenchymal transition in liver cancer cells. Additionally, the TP53INP1 expression level is inversely correlated with positive lymph node metastasis in clinical breast carcinoma tissue specimens (36-39). TP53INP1 suppresses pancreatic cancer cell migration by regulating the expression of SPARC, which is a matrix cellular protein that has a pivotal role in regulating cell-matrix interaction and migration (40). The expression of SPARC was increased and osteosarcoma cells were more aggressive after the miR-504 mimic transfection in vitro and the lung metastasis model confirmed the strengthened invasion ability of the miR-504 expressing cells in the present study.

Concerning limitations to the present study the use of miR-504 as a biomarker to predict the prognosis of early-stage osteosarcoma requires further validation with a larger sample size. The physical interaction between P73 and the miR-504/TP53INP1 axis warrants further exploration using rescue experiments with miR-504-resistant TP53INP1-plasmid in 143B cells, analysis using a yeast two-hybrid system and co-immunoprecipitation.

In conclusion, miR-504 directly targets TP53INP1 in osteosarcoma 143B cells. miR-504 promotes the proliferation and invasion of 143B cells and mitigates their apoptosis by targeting TP53INP1. As a result, P73 plays a role in tumour suppression independently of P53. The results of this study indicate that miR-504 and TP53INP1 expression are correlated with the tumour size and metastatic burden. 


\section{Acknowledgements}

This study is supported by the Shaanxi Provincial Health and Family Planning Commission Project (grant no. 2016D067) and the Foundation of the First Affiliated Hospital of Medical College, Xi'an Jiaotong University (grant no. 2013YK19; 2016MS-08).

\section{References}

1. Whelan J, McTiernan A, Cooper N, Wong YK, Francis M, Vernon S and Strauss SJ.: Incidence and survival of malignant bone sarcomas in England 1979-2007. Int J Cancer 131: E508-E517, 2012.

2. Savage SA and Mirabello L: Using epidemiology and genomics to understand osteosarcoma etiology. Sarcoma 2011: 548151, 2011.

3. Gill J, Ahluwalia MK, Geller D and Gorlick R: New targets and approaches in osteosarcoma. Pharmacol Ther 137: 89-99, 2013.

4. Ottaviani $\mathrm{G}$ and Jaffe N: The epidemiology of osteosarcoma. Cancer Treat Res 152: 3-13, 2009.

5. Pasquinelli AE, Hunter S and Bracht J: MicroRNAs: A developing story. Curr Opin Genet Dev 15: 200-205, 2005.

6. Zhou X and Yang PC: MicroRNA: A small molecule with a big biological impact. Microrna 1: 1, 2012.

7. Bartel DP: MicroRNAs: Genomics, biogenesis, mechanism, and function. Cell 116: 281-297, 2004.

8. Sassen S, Miska EA and Caldas C: MicroRNA - implications for cancer. Virchows Arch 452: 1-10, 2008.

9. Chang L, Shrestha S, LaChaud G, Scott MA and James AW: Review of microRNA in osteosarcoma and chondrosarcoma. Med Oncol 32: 613, 2015.

10. Sampson VB, Yoo S, Kumar A, Vetter NS and Kolb EA: MicroRNAs and potential targets in osteosarcoma: Review. Front Pediatr 3: 69, 2015.

11. Zhang J, Yan YG, Wang C, Zhang SJ, Yu XH and Wang WJ: MicroRNAs in osteosarcoma. Clinica Chimica Acta 444: 9-17, 2015.

12. Cui R, Guan Y, Sun C, Chen L, Bao Y, Li G, Qiu B, Meng X, Pang $C$ and Wang Y: A tumor-suppressive microRNA, miR-504, inhibits cell proliferation and promotes apoptosis by targeting FOXP1 in human glioma. Cancer Lett 374: 1-11, 2016

13. Zhao L, Tang M, Hu Z, Yan B, Pi W, Li Z, Zhang J, Zhang L, Jiang W, Li G, et al: miR-504 mediated down-regulation of nuclear respiratory factor 1 leads to radio-resistance in nasopharyngeal carcinoma. Oncotarget 6: 15995-16018, 2015.

14. Kikkawa N, Kinoshita T, Nohata N, Hanazawa T, Yamamoto N, Fukumoto I, Chiyomaru T, Enokida H, Nakagawa M, Okamoto Y, et al: microRNA-504 inhibits cancer cell proliferation via targeting CDK6 in hypopharyngeal squamous cell carcinoma. Int J Oncol 44: 2085-2092, 2014.

15. Hu W, Chan CS, Wu R, Zhang C, Sun Y, Song JS, Tang LH, Levine AJ and Feng Z: Negative regulation of tumor suppressor p53 by microRNA miR-504. Mol Cell 38: 689-699, 2010.

16. Saadi H, Seillier M and Carrier A: The stress protein TP53INP1 plays a tumor suppressive role by regulating metabolic homeostasis. Biochimie 118: 44-50, 2015.

17. Tomasini R, Samir AA, Carrier A, Isnardon D, Cecchinelli B, Soddu S, Malissen B, Dagorn JC, Iovanna JL and Dusetti NJ: TP53INP1s and homeodomain-interacting protein kinase-2 (HIPK2) are partners in regulating p53 activity. J Biol Chem 278: 37722-37729, 2003.

18. Enneking WF, Spanier SS and Goodman MA: A system for the surgical staging of musculoskeletal sarcoma. 1980. Clin Orthop Relat Res 415: 4-18, 2003.

19. Livak KJ and Schmittgen TD: Analysis of relative gene expression data using real-time quantitative PCR and the 2(-Delta Delta C(T)) method. Methods 25: 402-408, 2001.

20. Inukai S and Slack F: MicroRNAs and the genetic network in aging. J Mol Biol 425: 3601-3608, 2013.

21. Yang MH, Lin BR, Chang CH, Chen ST, Lin SK, Kuo MY, Jeng YM, Kuo ML and Chang CC: Connective tissue growth factor modulates oral squamous cell carcinoma invasion by activating a miR-504/FOXP1 signalling. Oncogene 31: 2401-2411, 2012
22. Soutto M, Chen Z, Saleh MA, Katsha A, Zhu S, Zaika A, Belkhiri $A$ and El-Rifai W: TFF1 activates p53 through down-regulation of miR-504 in gastric cancer. Oncotarget 5: 5663-5673, 2014

23. Jiang B, Gu Y and Chen Y: Identification of novel predictive markers for the prognosis of pancreatic ductal adenocarcinoma. Cancer Invest 32: 218-225, 2014.

24. Baek D, Villén J, Shin C, Camargo FD, Gygi SP and Bartel DP: The impact of microRNAs on protein output. Nature 455: 64-71, 2008.

25. Xu J, Li CX, Li YS, Lv JY, Ma Y, Shao TT, Xu LD, Wang YY, Du L, Zhang YP, et al: MiRNA-miRNA synergistic network: Construction via co-regulating functional modules and disease miRNA topological features. Nucleic Acids Res 39: 825-836, 2011.

26. D'Orazi G, Cecchinelli B, Bruno T, Manni I, Higashimoto Y, Saito S, Gostissa M, Coen S, Marchetti A, Del Sal G, et al: Homeodomain-interacting protein kinase-2 phosphorylates p53 at Ser 46 and mediates apoptosis. Nat Cell Biol 4: 11-19, 2002.

27. Hofmann TG, Möller A, Sirma H, Zentgraf H, Taya Y, Dröge W, Will $\mathrm{H}$ and Schmitz ML: Regulation of p53 activity by its interaction with homeodomain-interacting protein kinase-2. Nat Cell Biol 4: 1-10, 2002.

28. Tomasini R, Seux M, Nowak J, Bontemps C, Carrier A, Dagorn JC, Pébusque MJ, Iovanna JL and Dusetti NJ: TP53INP1 is a novel p73 target gene that induces cell cycle arrest and cell death by modulating p73 transcriptional activity. Oncogene 24: 8093-8104, 2005.

29. Pflaum J, Schlosser S and Müller M: p53 family and cellular stress responses in cancer. Front Oncol 4: 285, 2014.

30. Dötsch V, Bernassola F, Coutandin D, Candi E and Melino G: p63 and p73, the ancestors of p53. Cold Spring Harb Perspect Biol 2: a004887, 2010.

31. Yoon MK, Ha JH, Lee MS and Chi SW: Structure and apoptotic function of p73. BMB Rep 48: 81-90, 2015.

32. Satija YK and Das S: Tyr99 phosphorylation determines the regulatory milieu of tumor suppressor p73. Oncogene 35: 513-527, 2016.

33. Gonzalez S, Prives C and Cordon-Cardo C: p73alpha regulation by Chk1 in response to DNA damage. Mol Cell Biol 23: 8161-8171, 2003.

34. Ferraris VA, Brown JR, Despotis GJ, Hammon JW, Reece TB, Saha SP, Song HK, Clough ER, Shore-Lesserson LJ, Goodnough LT, et al; Society of Thoracic Surgeons Blood Conservation Guideline Task Force; Society of Cardiovascular Anesthesiologists Special Task Force on Blood Transfusion; International Consortium for Evidence Based Perfusion: 2011 update to the Society of Thoracic Surgeons and the Society of Cardiovascular Anesthesiologists blood conservation clinical practice guidelines. Ann Thorac Surg 91: 944-982, 2011.

35. Mantovani F, Piazza S, Gostissa M, Strano S, Zacchi P, Mantovani R, Blandino G and Del Sal G: Pin1 links the activities of $\mathrm{c}-\mathrm{Abl}$ and $\mathrm{p} 300$ in regulating p73 function. Mol Cell 14: 625-636, 2004

36. Jiang F, Liu T, He Y, Yan Q, Chen X, Wang H and Wan X: MiR-125b promotes proliferation and migration of type II endometrial carcinoma cells through targeting TP53INP1 tumor suppressor in vitro and in vivo. BMC Cancer 11: 425, 2011.

37. Li Q, Han Y, Wang C, Shan S, Wang Y, Zhang J and Ren T: MicroRNA-125b promotes tumor metastasis through targeting tumor protein 53-induced nuclear protein 1 in patients with non-small-cell lung cancer. Cancer Cell Int 15: 84, 2015.

38. Liu F, Kong X, Lv L and Gao J: TGF- $\beta 1$ acts through miR-155 to down-regulate TP53INP1 in promoting epithelial-mesenchymal transition and cancer stem cell phenotypes. Cancer Lett 359: 288-298, 2015

39. Ito Y, Motoo Y, Yoshida H, Iovanna JL, Takamura Y, Miya A, Kuma K and Miyauchi A: Decreased expression of tumor protein p53-induced nuclear protein 1 (TP53INP1) in breast carcinoma. Anticancer Res 26 (6B): 4391-4395, 2006.

40. Seux M, Peuget S, Montero MP, Siret C, Rigot V, Clerc P, Gigoux V, Pellegrino E, Pouyet L, N'Guessan P, et al: TP53INP1 decreases pancreatic cancer cell migration by regulating SPARC expression. Oncogene 30: 3049-3061, 2011. 